\title{
Advanced Synthesis of the DELTA Parallel Robot for a Specified Workspace
}

\author{
M.A. Laribi ${ }^{1}$, L. Romdhane ${ }^{1^{*}}$ and S. Zeghloul ${ }^{2}$ \\ Laboratoire de Génie Mécanique, LAB-MA-05 \\ Ecole Nationale d'Ingénieurs de Sousse, Sousse 40031, \\ Laboratoire de Mécanique des Solides, UMR 6610 \\ Bd Pierre et Marie Curie, BP 30179,Futuroscope 86962 Chasseneuil $^{2}$ \\ Tunisia ${ }^{1}$ \\ France $^{2}$
}

\section{Introduction}

Parallel manipulators have numerous advantages in comparison with serial manipulators: Higher stiffness, and connected with that a lower mass of links, the possibility of transporting heavier loads, and higher accuracy. The main drawback is, however, a smaller workspace. Hence, there exists an interest for the research concerning the workspace of manipulators.

Parallel architectures have the end-effector (platform) connected to the frame (base) through a number of kinematic chains (legs). Their kinematic analysis is often difficult to address.

The analysis of this type of mechanisms has been the focus of much recent research. Stewart presented his platform in 1965 [1]. Since then, several authors [2],[3] have proposed a large variety of designs.

The interest for parallel manipulators (PM) arises from the fact that they exhibit high stiffness in nearly all configurations and a high dynamic performance. Recently, there is a growing tendency to focus on parallel manipulators with 3 translational DOF $[4,5,8,9,10$, $11,12,13$,$] . In the case of the three translational parallel manipulators, the mobile platform$ can only translate with respect to the base. The DELTA robot (see figure 1) is one of the most famous translational parallel manipulators [5,6,7]. However, as most of the authors mentioned above have pointed out, the major drawback of parallel manipulators is their limited workspace. Gosselin [14], separated the workspace, which is a six dimensional space, in two parts : positioning and orientation workspace. He studied only the positioning workspace, i.e., the region of the three dimensional Cartesian space that can be attained by a point on the top platform when its orientation is given. A number of authors have described the workspace of a parallel mechanism by discretizing the Cartesian workspace. Concerning the orientation workspace, Romdhane [15] was the first to address the problem of its determination. In the case of 3-Translational DOF manipulators, the workspace is limited to

* Corresponding author. email :lotfi.romdhane@enim.rnu.tn

Source: Parallel Manipulators, Towards New Applications, Book edited by: Huapeng Wu, ISBN 978-3-902613-40-0, pp. 506, April 2008, I-Tech Education and Publishing, Vienna, Austria 
a region of the three dimensional Cartesian space that can be attained by a point on the mobile platform.

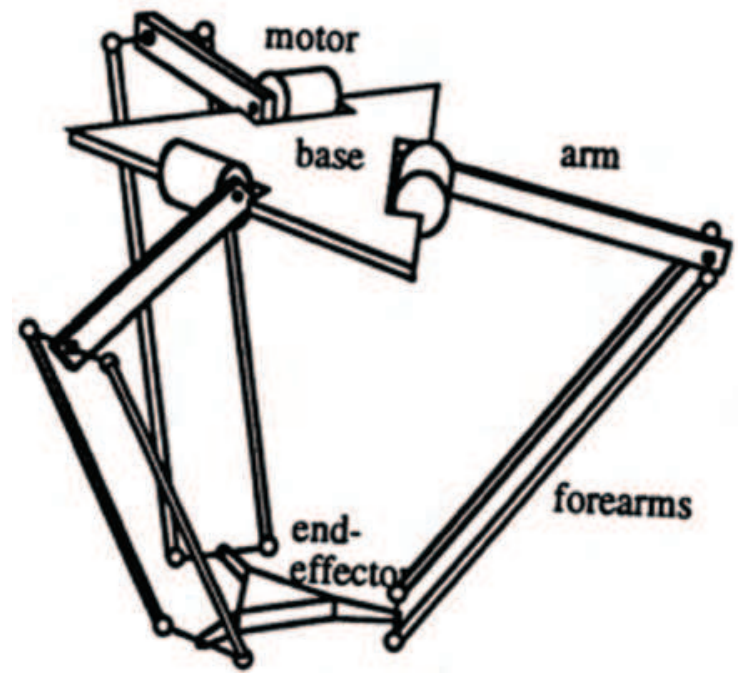

Fig. 1: DELTA Robot (Clavel R. 1986)

A more challenging problem is designing a parallel manipulator for a given workspace. This problem has been addressed by Boudreau and Gosselin [16,17], an algorithm has been worked out, allowing the determination of some parameters of the parallel manipulators using a genetic algorithm method in order to obtain a workspace as close as possible to a prescribed one. Kosinska et al. [18] presented a method for the determination of the parameters of a Delta-4 manipulator, where the prescribed workspace has been given in the form of a set of points. Snyman et al. [19] propose an algorithm for designing the planar 3RPR manipulator parameters, for a prescribed (2-D) physically reachable output workspace. Similarly in [20] the synthesis of 3-dof planar manipulators with prismatic joints is performed using GA, where the architecture of a manipulator and its position and orientation with respect to the prescribed worskpace were determined.

In this paper, the three translational DOF DELTA robot is designed to have a specified workspace. The genetic algorithm (GA) is used to solve the optimization problem, because of its robustness and simplicity.

This paper is organized as follows: Section 2 is devoted to the kinematic analysis of the DELTA robot and to determine its workspace. In Section 3, we carry out the formulation of the optimization problem using the genetic algorithm technique. Section 4 deals with the implementation of the proposed method followed by the obtained results. Finally, Section 5 contains some conclusions.

\section{Kinematic analysis and workspace of the DELTA robot}

\subsection{Direct and inverse geometric analyses}

The Delta robot consists of a moving platform connected to a fixed base through three parallel kinematic chains. Each chain contains a rotational joint activated by actuators in the 
base platform. The motion is transmitted to the mobile platform through parallelograms formed by links and spherical joints (See Figure 2).

We assume that all the 3 legs of the DELTA robot are identical in length. The geometric parameters of the DELTA robot are then given as: $\mathrm{L}_{1}, \mathrm{~L}_{2}, \mathrm{r}_{\mathrm{A}}, \mathrm{r}_{\mathrm{B}}, \theta_{j}$ for $j=1,2,3$ defined in Figure 2, as well as $\varphi_{1 j}, \varphi_{2 j}, \varphi_{3 j}$ for $j=1,2,3$ the joint angles defining the configuration of each leg. Let $P$ be a point lacated on the moving plateform, the geometric model can be written as :

$$
\begin{aligned}
X_{P}= & X_{A}+\cos \theta_{j}\left(L_{2} \cos \varphi_{1 j}+L_{1} \cos \varphi_{3} \cos \left(\varphi_{1 j}+\varphi_{2 j}\right)-r_{A}\right) \\
& -L_{1} \sin \theta_{j} \sin \varphi_{3 j}
\end{aligned}
$$

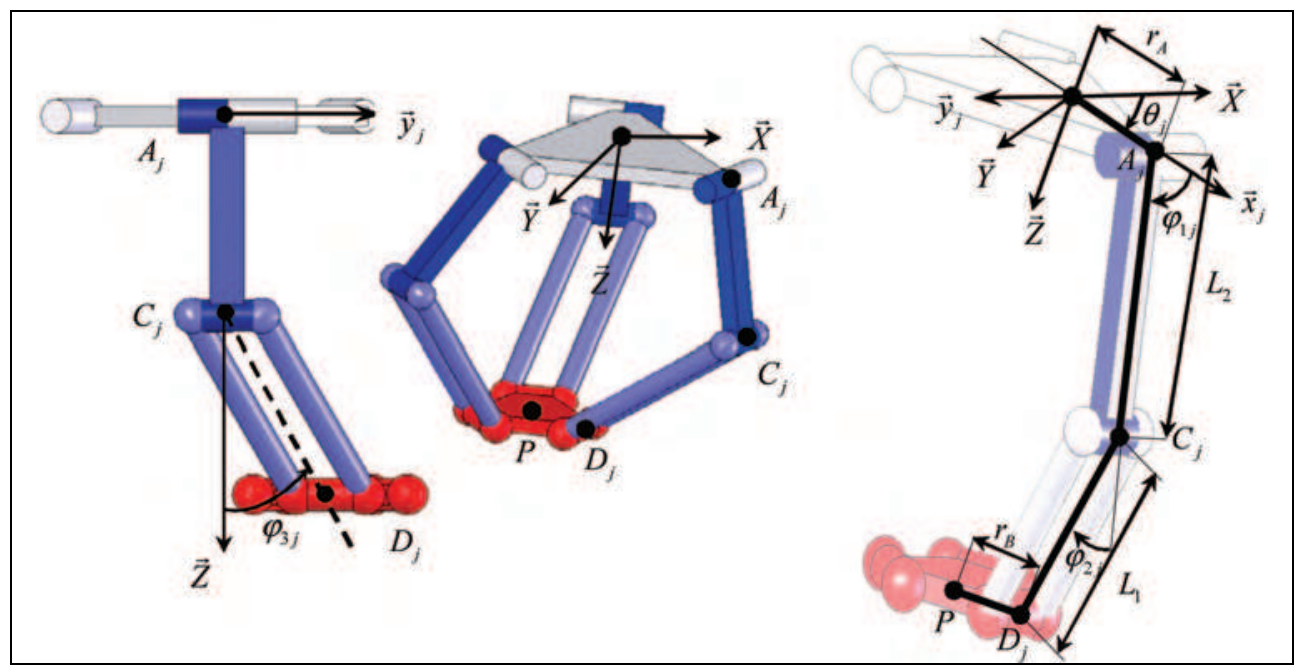

Fig. 2: The DELTA robot parameters.

$$
\begin{aligned}
& Y_{P}=Y_{A}+\sin \theta_{j}\left(L_{2} \cos \varphi_{1 j}+L_{1} \cos \varphi_{3 j} \cos \left(\varphi_{1 j}+\varphi_{2 j}\right)-r_{A}\right) \\
& +L_{1} \cos \theta_{j} \sin \varphi_{3 j} \\
& Z_{P}=L_{2} \sin \varphi_{1 j}+L_{1} \cos \varphi_{3 j} \sin \left(\varphi_{1 j}+\varphi_{2 j}\right)
\end{aligned}
$$

With $j=1, . ., 3$

Where $\left[X_{P} Y_{P} Z_{P}\right]$ are the coordinates of the point $P$.

In order to eliminate the passive joint variables we square and add these equations, which yields :

$$
\begin{array}{r}
{\left[\left(r+L_{2} \cos \varphi_{1 j}\right) \cos \theta_{j}-X_{P}\right]^{2}+\left[\left(r+L_{2} \cos \varphi_{1 j}\right) \sin \theta_{j}-Y_{P}\right]^{2}} \\
+\left[-L_{2} \cos \theta_{j}-Z_{P}\right]^{2}-L_{1}^{2}=0
\end{array}
$$


Where $j=1, . ., 3$ and $r=r_{A}-r_{B}$.

\subsubsection{The direct geometric model}

The direct problem is defined by (4), where the unknowns are the location of point $P=\left[X_{\mathrm{p}}\right.$ $\left.Y_{p}, Z_{p}\right]$ for a given joint angles $\varphi_{1 j}, \varphi_{2 j}, \varphi_{3 j}(j=1, . ., 3)$.

This equation can be put in the following form:

$$
\left(X_{P}-X_{j}\right)^{2}+\left(Y_{P}-Y_{j}\right)^{2}+\left(Z_{P}-Z_{j}\right)^{2}=L_{1}^{2}
$$

where,

$$
\left\{\begin{array}{c}
X_{j}=r+L_{2} \cos \varphi_{1 j} \\
Y_{j}=r+L_{2} \cos \varphi_{1 j} \\
Z_{j}=-L_{2} \cos \theta_{j}
\end{array}\right.
$$

Equation (5) represents a sphere centred in point $S_{j}\left[X_{j}, Y_{j}, Z_{j}\right]$ and with radius $L_{1}$.

The solution of this system of equations can be represented by a point defined as the intersection of these three spheres. In general, there are two possible solutions, which means that, for a given leg lengths, the top platform can have two possible configurations with respect to the base. For more details see ref [21].

\subsubsection{Inverse geometric model}

The inverse problem is defined by (4), where the unknowns are the joint angles $\varphi_{1 j}, \varphi_{2 j}, \varphi_{3 j}$ $(j=1,2,3)$ for a given location of the point $P=\left[X_{P}, Y_{P}, Z_{P}\right]$.

$$
\begin{aligned}
& \left(2 r L_{2}-2 L_{2} X_{P} \cos \theta_{j}-2 L_{2} Y_{P} \sin \theta_{j}\right) \cos \varphi_{1 j}-2 r X_{P} \cos \theta_{j}+2 L_{2} Z_{P} \sin \varphi_{1 j} \\
& -2 r Y_{P} \sin \theta_{j}+X_{P}^{2}+r^{2}+L_{2}^{2}+Z_{P}^{2}+Y_{P}^{2}-L_{1}^{2}=0
\end{aligned}
$$

which can be written as:

$$
l_{j} \cos \varphi_{1 j}+m_{j} \sin \varphi_{1 j}-n_{j}=0
$$

Where,

$$
\left\{\begin{array}{l}
l_{j}=2 r L_{2}-2 L_{2} X_{P} \cos \theta_{j}-2 L_{2} Y_{P} \sin \theta_{j} \\
m_{j}=2 L_{2} Z_{P} \\
n_{j}=-2 r X_{P} \cos \theta_{j}-2 r Y_{P} \sin \theta_{j}+X_{P}^{2}+r^{2}+L_{2}^{2}+Z_{P}^{2}+Y_{P}^{2}-L_{1}^{2}
\end{array}\right.
$$

Equation (8) can have a solution if and only if:

$$
\left|\frac{n_{j}}{\sqrt{l_{j}^{2}+m_{j}^{2}}}\right| \leq 1 \Leftrightarrow n_{j}^{2}-\left(l_{j}^{2}+m_{j}^{2}\right) \leq 0
$$


For more details on the inverse geometric model of the DELTA robot see [21,22,23].

\subsection{Workspace of the DELTA robot}

The workspace of the DELTA robot is defined as a region of the three-dimensional cartesian space that can be attained by a point on the platform where the only constraints taken into account are the ones coming from the different chains given by Equations (10). Equation (10) can be written as:

$$
\begin{gathered}
h_{j}\left(X_{P}, Y_{P}, Z_{P}\right)=\left(\begin{array}{c}
\left(X_{P} \cos \theta_{j}+Y_{P} \sin \theta_{j}-r\right)^{2} \\
+\left(X_{p} \sin \theta_{j}-Y_{P} \cos \theta_{j}\right)^{2}+Z_{P}^{2}+L_{2}^{2}-L_{1}^{2}
\end{array}\right)^{2} \\
-4 L_{2}^{2}\left(\left(X_{P} \cos \theta_{j}+Y_{P} \sin \theta_{j}-r\right)^{2}+Z_{P}^{2}\right) \leq 0
\end{gathered}
$$

Equation (11) in cartesian coordinates for a torus azimuthally symmetric about the $y$-axis can be writen as follows :

$$
\left((x-r)^{2}+y^{2}+z^{2}+a^{2}-b^{2}\right)=4 d^{2}\left(x^{2}+z^{2}\right)
$$

Where, $a=L_{2}$ and $b=L_{1}$

The set of points $P$ satisfying $h_{j}\left(X_{P}, Y_{P}, Z_{P}\right)=0$ are the ones located on the boundary of this workspace. This volume is actually the result of the intersection of three tori. Each torus is centered in point $O_{j}\left(r \cos \theta_{j}, r \sin \theta_{j}, 0\right)$ and with a minor radius given by $L_{2}$ and a major radius given by $L_{1}$. Figure 3 shows the upper halves of these tori. In the following, we will be interested only in the upper half of the workspace.

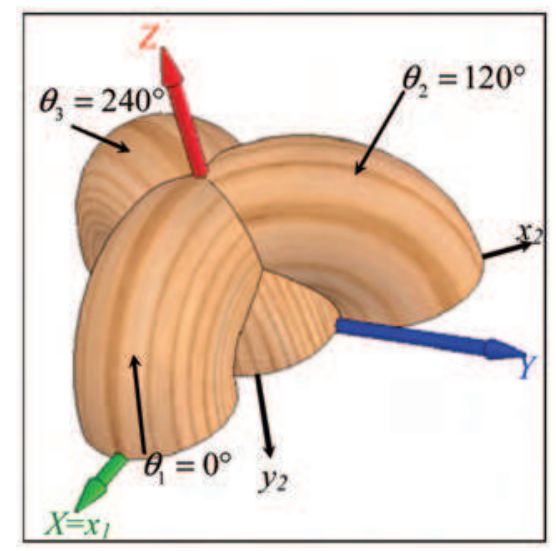

Fig. 3: The three upper halves of the tori given by $h_{j}(P)=0$

Therefore, one can state that for a given point $P\left(X_{P}, Y_{P}, Z_{P}\right)$ :

if $P$ is inside the workspace then $h_{j}(P)<0$ for $j=1,2,3$.

if $P$ is on the boundary of the workspace then $h_{j}(P) \leq 0$ for $j=1,2,3$ and $h_{j}(P)=0$ for $j=1$ or $j=2$ or $j=3$.

if $P$ is outside the workspace then $h_{j}(P)>0$ for $j=1$ or $j=2$ or $j=3$. 


\section{Dimensional synthesis of the DELTA robot for a given workspace}

\subsection{Formulation of the problem}

The aim of this section is to develop and to solve the multidimensional, non linear optimization problem of selecting the geometric design variables for the DELTA robot having a specified workspace. This specified workspace has to include a desired volume in space, $\mathrm{W}$. This approach is based on the optimization of an objective function using the genetic algorithm (GA) method.

The dimensional synthesis of the DELTA robot for a given workspace can be defined as follows:

Given : a specified volume in space W.

Find : the smallest dimensions of the DELTA robot having a workspace that includes the specified volume.

For example if the specified volume is a cube, then the workspace of the DELTA robot has to include the given cube.

The optimization problem can be stated as:

$\min F(I)$

Subject to

$\mathrm{h}_{j}(\mathrm{I}, \mathrm{P}) \leq 0$ for all the points $\mathrm{P}$ inside the specified volume $\mathrm{W}$.

$$
\begin{gathered}
x_{i} \in I \\
x_{i} \in\left[x_{i \min }, x_{i \max }\right]
\end{gathered}
$$

$h_{j}$ : are the constraints applied on the system.

$I:$ is a vector containing the independent design variables.

$x_{i}$, is an element of the vector $I$, called individual in the genetic algorithm technique.

$x_{i \min }$ and $x_{i \max }$ are the range of variation of each design variable.

If the volume can be defined by a set of vertices $P_{k}\left(k=1, N_{p t}\right)$, then the desired volume $\mathrm{W}$ is inside the workspace of the DELTA robot if:

$$
h_{j}\left(I, P_{k}\right) \leq 0, j=1, . ., 3 \text { and } k=1, . ., N p t
$$

In this work, we will take the case where $\mathrm{W}$ is a cube given by $N_{p t}=8$ points (see Figure 4 ). For every workspace to be generated by a DELTA robot, the independent design variables are:

$$
h_{j}\left(I, P_{k}\right) \leq 0, j=1, . ., 3 \text { and } k=1, . ., N p t
$$

Where $H$ is a parameter defining how far is the specified volume from the base of the DELTA robot (see Figure 4). This function $h_{j}$ when applied to a point can be used as a measure of some kind of distance of this point with respect to the surface defined by $h_{j}=0$. In geometry, this function is called the power of the point with respect to the surface. In the plane, $h_{j}=0$ defines a curve. Annex I presents some theoretical background about the power of a point with respect to a circle. Moreover, the function $h_{j}$ changes its sign depending on which side of the surface the point is located. Therefore minimizing the function $\left|h_{j}(P)\right|$, is 
equivalent to finding the closest point to the given surface. In our case, we are looking for a volume bounded by three surfaces, therefore one has to minimize the function $f=\left|h_{1}(I, P)\right|$ $+\left|h_{2}(I, P)\right|+\left|h_{3}(I, P)\right|$. Figure 5 represents a mapping, $f(x, y)$, of the power of points at a given height $z_{0}=1$ as a function of $x$ and $y$ for a given design vector $I=[1.9,1.2,0.9,1]$.

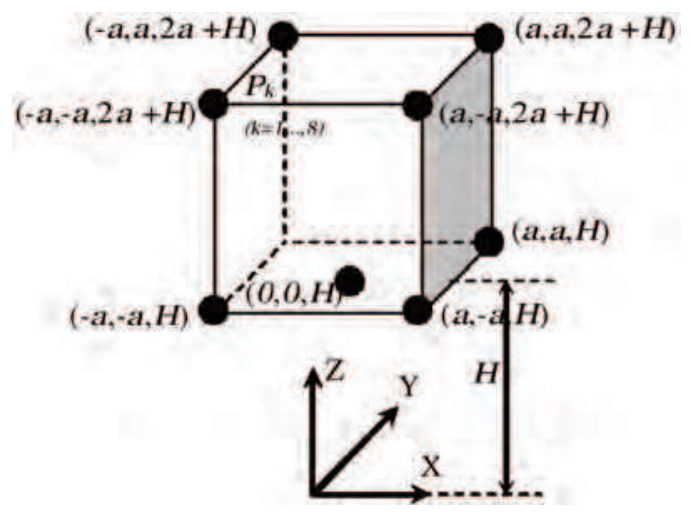

Fig. 4: The scheme of the prescribed workspace.

The function $f$ is given by:

$$
f=\left|h_{1}(I, P)\right|+\left|h_{2}(I, P)\right|+\left|h_{3}(I, P)\right|
$$

One can notice that the minimum value of $f$ is obtained when the point is located on the boundary of the workspace (see Figure 5).

Our objective is to find the smallest set of parameters, given by $I=\left[L_{1}, L_{2}, r, H\right]$ that can yield a DELTA robot having a workspace that includes the given volume in space $W$.

The methodology followed to solve this problem is based on minimizing the power of the vertices, defining the given volume, and to ensure that all these vertices have a negative power, i.e., they are inside the workspace of the DELTA robot. This minimization problem will be solved using the GA method.

It is worth noting that this procedure is valid for any convex volume defined by a set of vertices.

\subsection{GA optimization}

The GA is a stochastic global search method that mimics the metaphor of natural biological evolution [24]. GAs operate on a population of potential solutions applying the principle of survival of the fittest to produce better and better approximations to a solution. At each generation, a new set of approximations is created by the process of selecting individuals according to their level of fitness in the problem domain and breeding them together using operators borrowed from natural genetics. This process leads to the evolution of populations of individuals that are better suited to their environment than the individuals that they were created from, just as in natural adaptation. The GA differs substantially from more traditional search and optimization methods. The four most significant differences are:

- GAs search a population of points in parallel, not a single point. 
- GAs do not require derivative information or other auxiliary knowledge; only the objective function and corresponding fitness levels influence the directions of search.

- GAs use probabilistic transition rules, not deterministic ones.

- A number of potential solutions are obtained for a given problem and the choice of final solution can be made, if necessary, by the user.

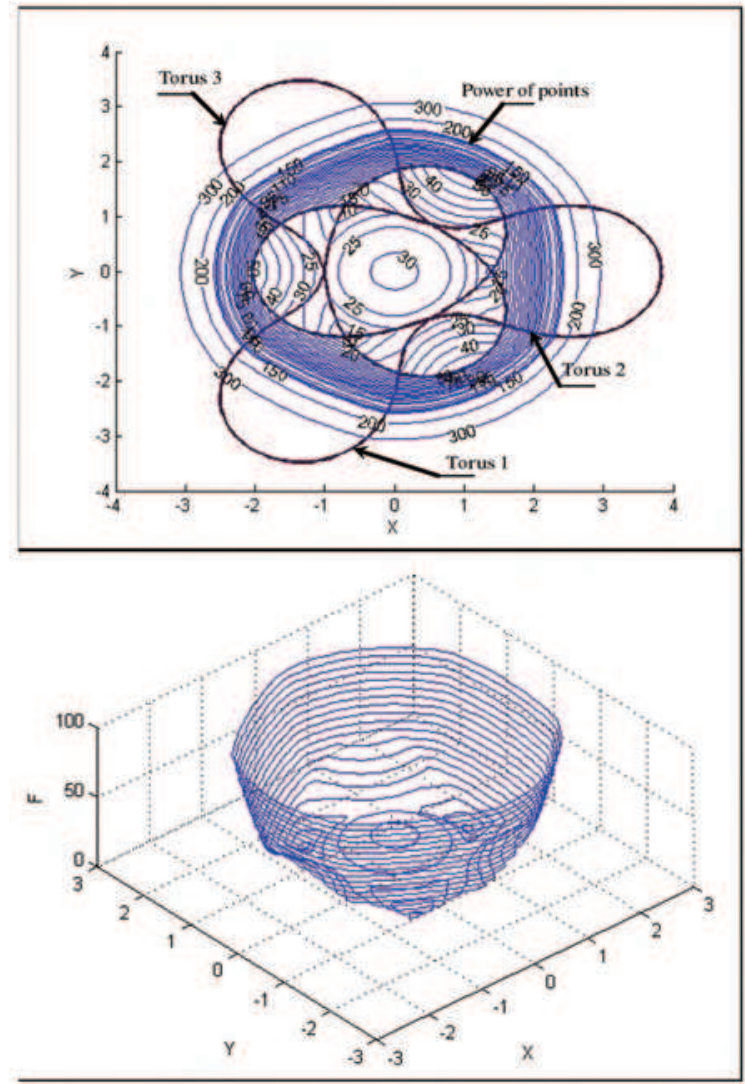

Fig. 5: Graphical representation of the power of a point $F(X, Y)$.

In most applications involving GAs, binary coding is used. However,Wright [32] showed that real-coded GAs have a better performance than binary-coded GAs [25,26,27,28,29]. A real-coded GA is used in this work. The description of the operations necessary for this type of code are presented by Figure 6, more details can be found in [30]. The parameters used in this work are shown in Table 1.

A penalty function method is used to handle the constraints and to ensure that the fitness of any feasible solution is better than infeasible ones.

The fitness function is constructed as:

$$
\digamma=-\left(\digamma_{1}+\digamma_{2}\right)
$$


Where $F_{1}$ is a penality function defined as follows:

$$
\digamma_{1}=\sum_{k=1}^{N p t} \sum_{j=1}^{3} \wp_{j}\left(I, P_{k}\right)
$$

where

$$
\wp_{j}\left(I, P_{k}\right)=\left\{\begin{array}{cll}
0 & \text { if } & h_{j}\left(I, P_{k}\right) \leq 0 \\
c f & \text { if } & h_{j}\left(I, P_{k}\right)>0
\end{array}\right.
$$

Where, $c f$ is a large positive constant.

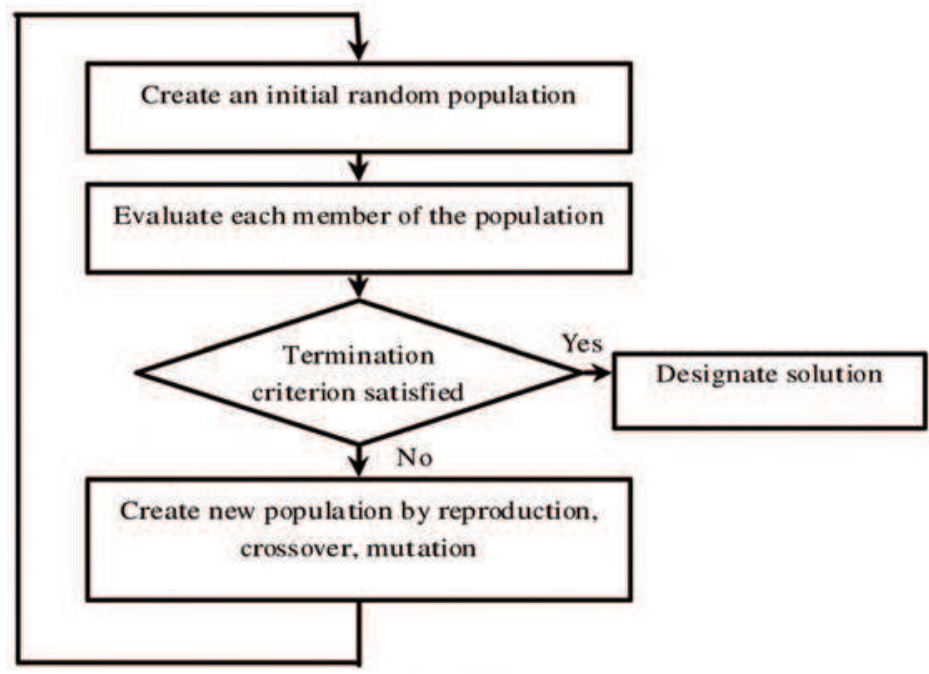

Fig. 6: Genetic algorithm flowchart.

\begin{tabular}{lll}
\hline The population size & $N_{\text {ind }}:$ & 100 \\
\hline The maximal generation number & $M_{a x} G_{e n}:$ & 100 \\
\hline The variable number & $N_{\text {var }}:$ & 4 \\
\hline The generation gap & $G_{g a p}:$ & 0.85 \\
\hline The mutation rate & $F_{1}:$ & 0.64 \\
\hline The shrinking mutation factor & $F_{2}:$ & 0.615 \\
\hline The recombination rate & $\mathrm{Opt}_{1}:$ & 0.623 \\
\hline The shrinking recombination factor & $\mathrm{Opt}:$ & 0.553 \\
\hline
\end{tabular}

Tab. 1: Parameters used for the genetic algorithm.

$F_{1}=0$ means that all the vertices defining the volume $\mathrm{W}$ are contained within the workspace of the DELTA robot. In this case, the fitness $F_{2}$ is given by

$$
\digamma_{2}=\sum_{k=1}^{N p t} \sum_{j=1}^{3}\left|h_{j}\left(I, P_{k}\right)\right|
$$


In the case when $F_{1} \neq 0$, i.e., at least one of the vertices is outside the workspace, $F_{2}$ is set to zero $\left(F_{2}=0\right)$.

\section{Results}

All the results, presented in this section, are obtained on a Pentium M processor of $1500 \mathrm{Mhz}$ and the programs are developed under MATLAB. The calculation time, necessary for obtaining the optimum solution, is estimated at about $4 \mathrm{~s}$.

\subsection{Example 1}

In this example, the dimensions of the DELTA robot are to be determined to get the smallest workspace capable of containing a volume $W$, given by a cube with a side $2 a=2$ (Figure 4 ). The bounding interval for each one of the design variables is presented in Table 2 :

\begin{tabular}{ccccc}
\hline$I$ & $L_{1}$ & $L_{2}$ & $r$ & $H$ \\
\hline$x_{\min }$ & 0.5 & 0.5 & 0 & 1 \\
\hline$x_{\max }$ & 5 & 5 & 5 & 5 \\
\hline
\end{tabular}

Tab. 2: The bounding interal for design variables

The optimal solution obtained by the GA for this example is presented in Table 3:

\begin{tabular}{ccccc}
\hline & $L_{1}$ & $L_{2}$ & $r$ & $H$ \\
\hline$I$ & 2.66 & 1.73 & 1.74 & 1.01 \\
\hline
\end{tabular}

Tab. 3: The optimal dimension of DELTA robot (example 1)

Figure 7 presents a mapping, $f$, of the power of points at a given height equal to 1.01 as a function of $x$ and $y$ for the optimal solution. A 3D representation of the platform and the corresponding workspace along with the desired volumeW, is shown on Figure 8. Figure 9 presents horizontal slices of the workspace at the lower and upper faces of the cube. One can notice that the upper vertices of the cube are exactly located on the boundary of the workspace; which means that the robot has to be in an extreme position (on the boundary of the workspace) to be able to reach these points. To avoid this problem, we propose to design a robot having a slightly bigger workspace defining this way a safety region. The following example illustrates this problem.

\subsection{Example 2}

In this second example, a distance is kept between the workspace of the DELTA robot and the desired volume. To have this safety region, we used the fact that a safety distance can be kept, during the optimization, between each vertex and the surface defining the boundary of the workspace. This safety distance can be translated in terms of the power of the point, which means that, during the optimization, a lower bound is set on the powers of all points. This lower bound ensures that in the final solution no point can be on the surface defining the boundary of the workspace, i.e., the power is zero in that case, but rather on a surface parallel to the boundary of the workspace. The distance between these two surfaces is defined as the safety distance. 


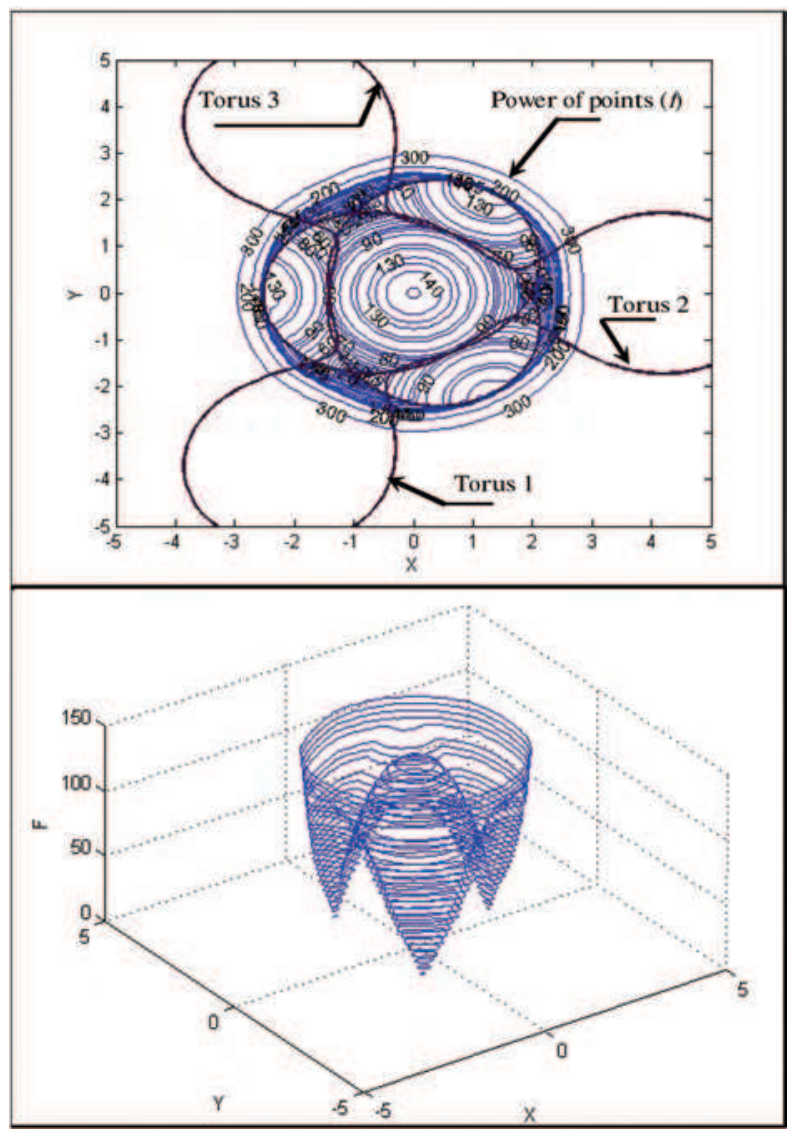

Fig. 7: Graphical representation of the power of a point $F(X, Y)$ (example 1).

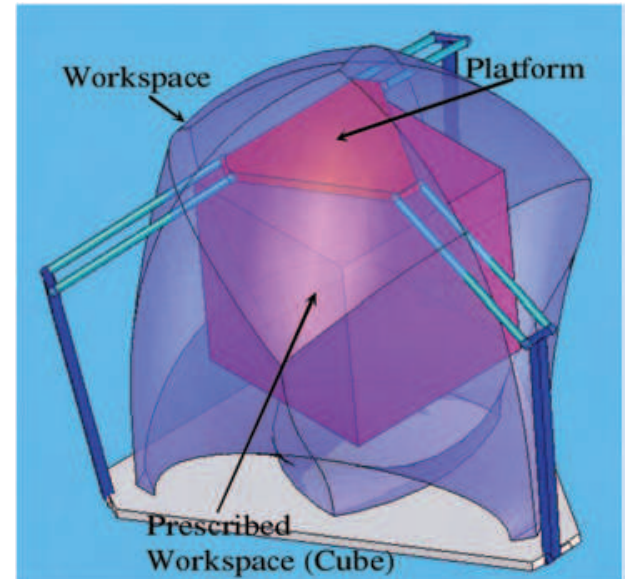

Fig. 8: The Optimal DELTA robot for example 1. 
In our case, the workspace is the intersection of three tori, each with $L_{1}$ as a major radius and $L_{2}$ as the minor radius. Therefore, the three corresponding tori, each with a major radius $L_{1}$ and a minor radius $L_{2}-e$, define a more restrictive volume of the workspace. The intersection of the smaller tori is now the bounding volume within which the desired volume $W$ has to be located. In this case, we took $e=0.1 L_{2}$.

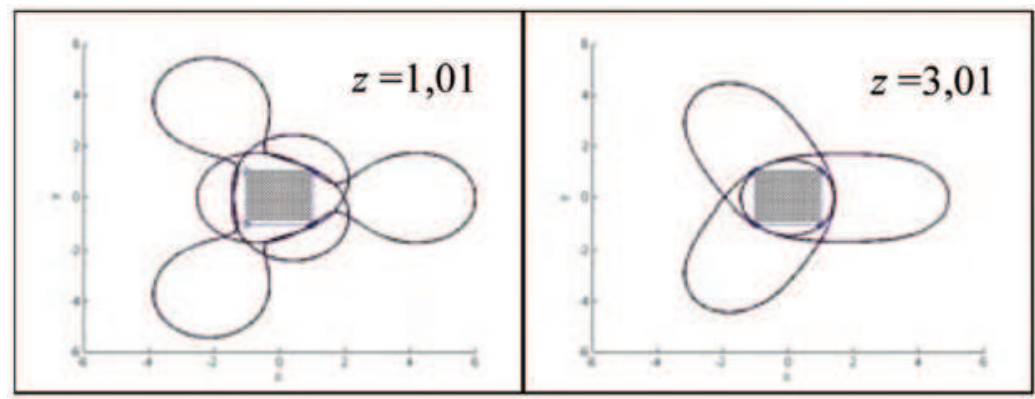

Fig. 9: Two slices of the workspace at the top and bottom of the cube.

The new optimal solution found for the DELTA robot is given by Table 4 . One can notice that $L_{1}$ and $r$ decreased, whereas $L_{2}$ increased, compared to the previous example. The height of the cube with respect to the base, $H$, stayed almost the same.

Figure 10 shows slices at the upper and lower faces of the cube of the workspace and the corresponding safety region. Figure 11 shows two cuts of the workspace with the cube inside it. One can notice that the vertices of the cube are kept at a minimum distance given by the safety distance $e$.

A 3D representation of the platform and the corresponding workspace along with the desired volume $W$, is shown on Figure 12. One can notice that all the points of the cube can be reached by the platform without reaching an extreme configuration as it was the case, in the previous example.

\begin{tabular}{ccccc}
\hline & $L_{1}$ & $L_{2}$ & $r$ & $H$ \\
\hline$I$ & 2.5 & 1.91 & 1.48 & 1.02 \\
\hline
\end{tabular}

Tab. 4: The optimal dimension of DELTA robot with safety zone (example 2)

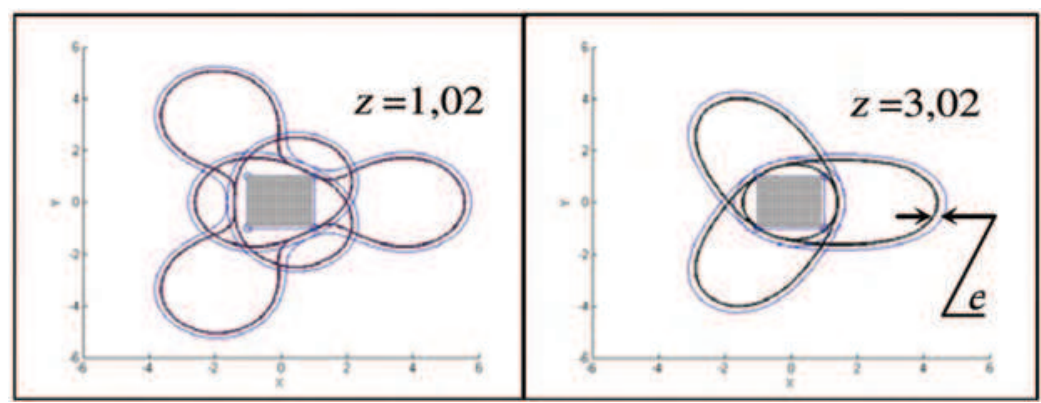

Fig. 10: Two slices of the workspace at the top and bottom of the cube with a safety zone. 

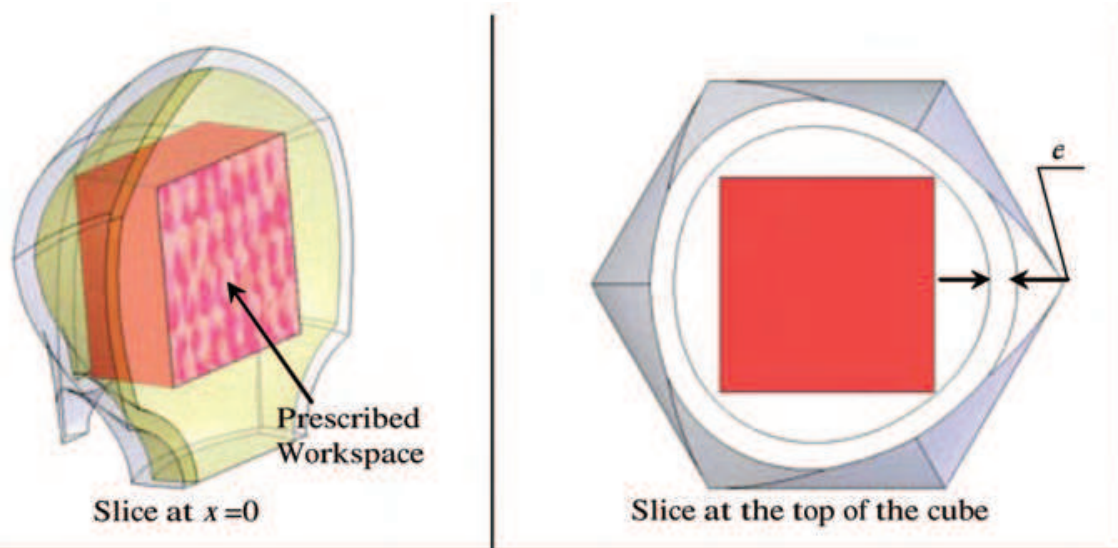

Fig. 11: Different slices of the Workspace.

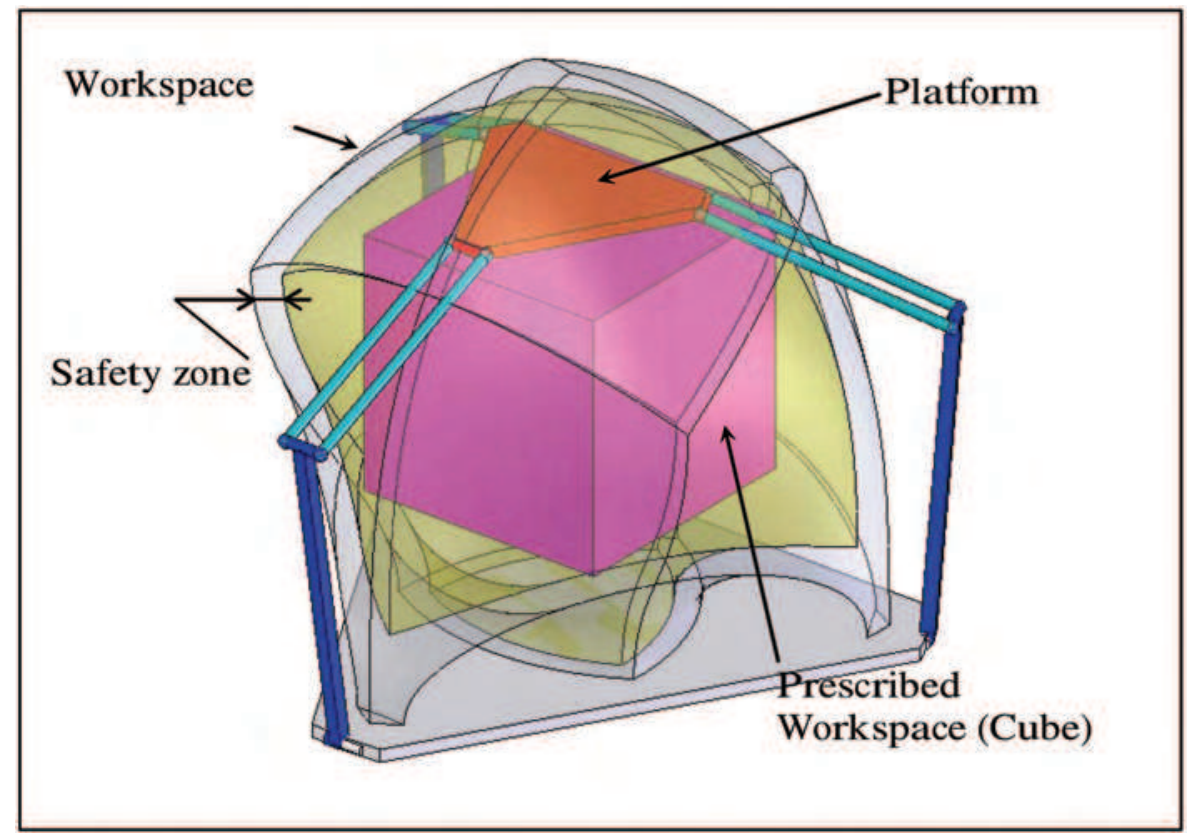

Fig. 12: The Optimal DELTA robot.

\subsection{Example 3}

In this example we propose an hexagonal prism as a prescribed workspace, given by $\mathbf{N}_{p t}=$ 13 points (see figure 13). The dimensions of the DELTA robot are to be determined to get the smallest workspace capable of containing a volume $\mathbf{W}$, given by an hexagonal prism with a side $\mathbf{b}=1$. The bounding interval for each one of the design variables is presented in Table 5: 


\begin{tabular}{ccccc}
\hline$I$ & $L_{1}$ & $L_{2}$ & $r$ & $H$ \\
\hline$x_{\min }$ & 0.5 & 0.5 & 0 & 1 \\
\hline$x_{\max }$ & 7 & 7 & 7 & 7 \\
\hline
\end{tabular}

Tab. 5: The bounding interval for design variables

Figure 14 and 15 present a mapping, $f$, of the power of a point at a given height equal to 1.67 as a function of $x$ and $y$ for the optimal solution obtained by the GA presented in Table 6 .

A 3D representation of the platform and the corresponding workspace along with the desired volume W, are shown on Figure 16.

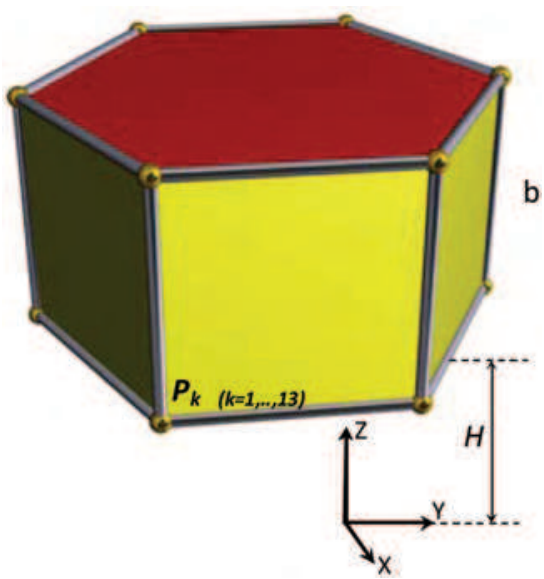

Fig. 13: The scheme of an hexagonal prism prescribed workspace.

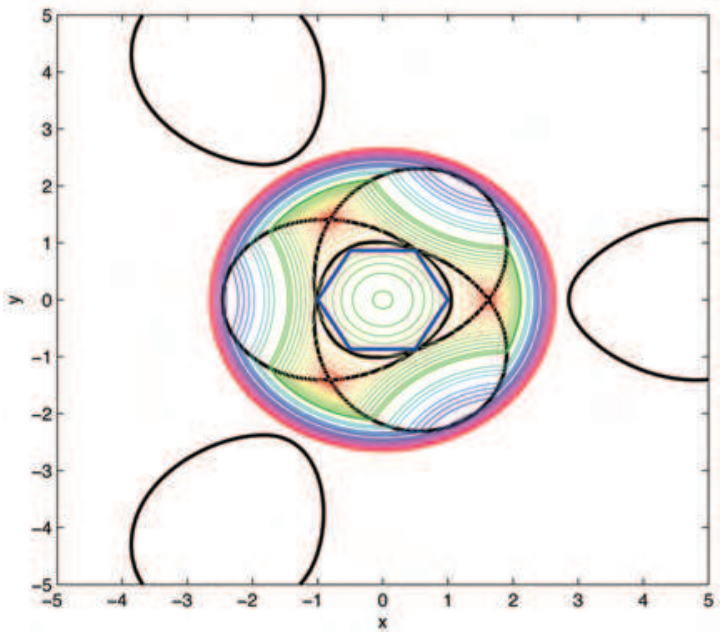

Fig. 14: Graphical representation of the power of a point $F(X, Y)$ for an hexagonal prism prescribed workspace. 


\begin{tabular}{ccccc}
\hline & $L_{1}$ & $L_{2}$ & $r$ & $H$ \\
\hline$I$ & 3.32 & 1.4 & 1.95 & 1.67 \\
\hline
\end{tabular}

Tab. 6: The optimal dimension of the DELTA robot with a hexagonal prism as a prescribed workspace

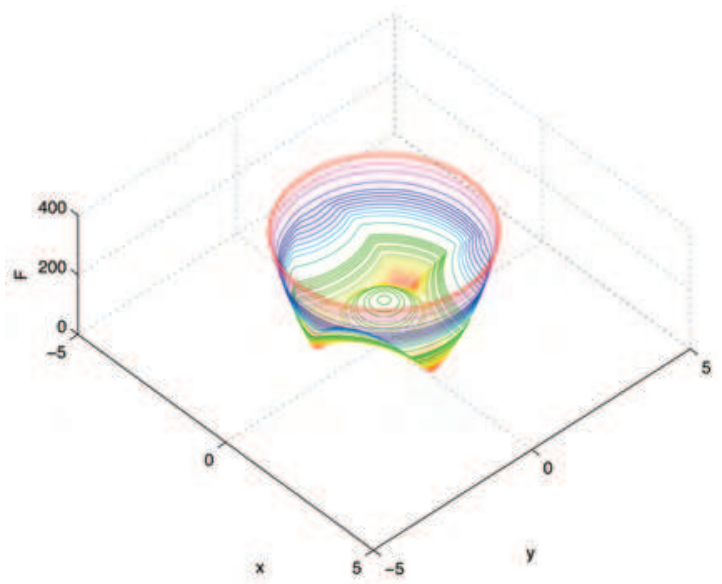

Fig. 15: Graphical representation of the power of a point $F(X, Y)$ for a hexagonal prism as a prescribed workspace.

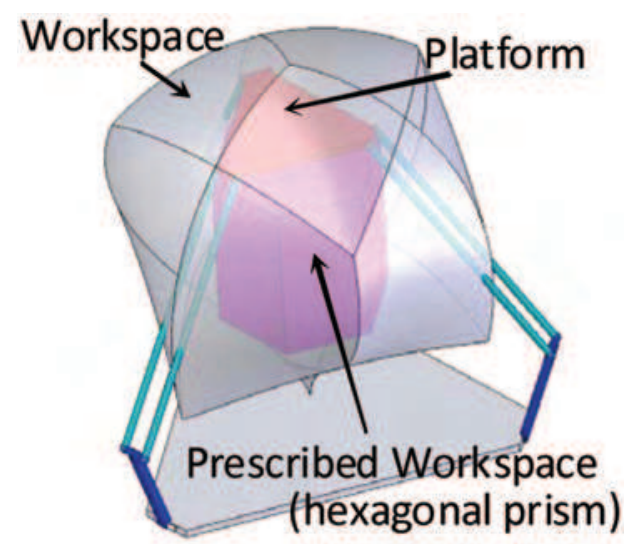

Fig. 16: The Optimal DELTA robot for an hexagonal prism as a prescribed workspace.

\section{Conclusion}

An optimal dimensional synthesis method suited for the DELTA robot was presente in this paper. An objective function, used the concept of the power of a point,which reflects the position of a point with respect to the boundary of the workspace. This objective function allowed us to find the robot having the smallest workspace containing a prespecified region. 
The genetic algorithm method was used.The prescribed region was chosen as a cube then as an hexagonal prism. The obtained solution yields a workspace where some of the vertices of the cube or the hexagonal prism are located on the boundary of the workspace. To reach these points the DELTA robot has to get into extreme configurations. To avoid this problem, we introduced a safety distance allowing us to have all the prespecified region inside the workspace. The concept of the power of a point along with the GA method turned out to be an effective and easy tool to solve the problem of designing a DELTA robot for a specified workspace. This method can also be applied, in a similar manner, to any convex prismatoid prespecified region of the workspace.

\section{References}

D. Stewart,1965, "A platform with 6 degrees of freedom", Proc. of the Institution of mechanical engineers, Vol. 180 (Part 1, 15),pp. 371-386, 1965.

E. F. Fichter,1986, "A Stewart platform based manipulator: general theory and practical construction",International Journalof Robotic Resarch, Vol. 5, pp. 157-182.

Griffis, M., and Duffy, J., "A Forward Displacement Analysis of a Class of Stewart Platforms," Trans. ASME Journal of Mechanisms, Transmissions, and A utomation in Design, Vol. 6, No. 6, June 1989, pp. 703-720.

Affi Z., Romdhane L. and Maalej A., 2004. Dimensional synthesis of a 3-translational-DOF in-parallel manipulator for a desired workspace. European Journal of Mechanics A/Solids, Vol 23, Issue 2, pp 311-324.

Clavel, R. 1986. Une nouvelle structure de manipulation parallèle pour la robotique légère. R.A.I.R.O. APII, $\mathrm{Vol} 23, \mathrm{~N}^{\circ} 6$.

Vischer P. and Clavel R. 1998,"Kinematic Calibration of the Parallel Delta Robot", Robotica, 16 , pp. 207-218.

M. Stock and K. Miller 2003, "Optimal Design of Spatial Parallel Manipulators: Application to linear Delta Robot", ASME Journal of Mechanical Design, Vol. 125, pp 292-301.

Hervé J. M. 1995, “Design of Parallel Manipulators via Displacement Group", Proceedings of the 9th World Congress on the Theory of Machines and Mechanisms. pp. 20792082.

Hervé, J. M., Sparacino F. 1991. Structural synthesis of parallel robots generating spatial translation. 5th Int.Conf. On Adv. Robotics, IEEE n91TH0367-4, Vol. 1, pp 808-813.

Romdhane, L. 1999, Design and analysis of a hybrid serial-parallel manipulator. Mechanism and Machine Theory, Vol. 34, Issue 7, pp 1037-1055.

Romdhane, L., Affi Z., Fayet M., 2002. Design and singularity analysis of a 3 translationalDOF in-parallel manipulator. ASME Journal of Mechanical Design, Vol. 124, pp 419-426.

A. Tremblain and L. Baron 1999, "Geomatrical synthesis of parallel manipulators of star-like topology with a geometric algorithm", IEEE International Conference on Robotics and Automation, Detroit, MI.

Tsai L-W 1996, "Kinematics of three-dof platform with three extensible limbs” In J. Lenarcic V. Parenti-Castelli, editor, Recent Advances in Robot Kinematics, pp 401 410, Kluwer.

C. Gosselin,1990, "Determination of the workspace of 6-dof parallel manipulators", ASME Journal of Mechanical Design, Vol. 112, pp. 331-336. 
L. Romdhane,1994, "Orientation workspace of fully parallel mechanisms", Eur. J. of Mechanics Vol. 13, pp. 541-553.

R. Boudreau and C. M. Gosselin 1999, "The synthesis of planar parallel manipulators with a genetic algorithm", ASME Journal of Mechanical Design, Vol 121, pp 533-537.

R. Boudreau and C. M. Gosselin 2001, "La synthèse d'une plate forme de Gough-Stewart pour un espace de travail atteignable prescrit", Mech. Mach. Theory 36 (2001) 327342.

Kosinska, A, Galicki, M. and Kedzior, K. 2003,"Design and optimization of parameters of Delta-4 Parallel Manipulator for a Given Workspace", Journal of Robotic Systems 20 (9), pp 539-548.

J. A. Snyman and A. M. Hay 2005, "Optimal synthesis for a continuos prescribed dexterity interval of 3-DOF parallel planar manipulator for different prescribed output workspaces", Proceeding of CK2005, 12th International Workshop on Computational Kinematics Cassino May 4-6.

M. Gallant and R. Boudreau 2002, "The synthesis of planar parammel manipulators with prismatic joints for an optimal, singularity-free workspace", Journal of Robotic Systems 19 (1), pp 13-24.

F. Pierrot, C. Reynau and A. Fourier 1990, "DELTA : a simple and efficient parallel robot", Robotica Vol. 8, pp 105-109.

Goudali, A. 1995. Contribution à l'étude d'un nouveau robot Parallèle 2- Delta à six degrés de liberté avec découplage. Thèse de doctorat Génie Mécanique L.M.S. Poitiers. France.

J.P. Lallemand, A. Goudali and S. Zeghloul, "The 6 - D.o.f. 2 - Delta parallel robot", Robotica Journal, Vol. 15, pp 407-416, 1997.

Goldberg, D.E., 1994, Genetic Algorithms in Search, Optimization, and Machine Learning, Addison-Wesley Publishing, Reading, MA.

Chipperfield A., Fleming P., Pohlheim H. and Fonseca C. 1994, "Genetic AlgorithmTOOLBOX user's Guide" Department of automatic control and systems engineering university of Sheffield version ( $\mathrm{v} 1.2)$

J.A. Lozano , P. Larranaga, M. Grana, F.X. Albizuri 1999, Genetic algorithms: bridging the convergence gap, Theoretical Computer Science Vol. 229, pp 11-22.

R. Chelouah, P. Siarry 2000, A continuous genetic algorithm designed for the global optimization of multimodal functions, Journal of Heuristics Vol. 6, pp. 191-213.

Schmitt L. M. 2001, Fundamental Study Theory of genetic algorithms, Theoretical Computer science $\mathrm{n}^{\circ} 259 \mathrm{pp} 1-61$.

Laine, R., Zeghloul, S., Ramirez, G., 2002, A Method based on a Genetic Algorithm for the Optimal Design of Serial Manipulators, Int. Symp. Rob. and Aut., pp. 15-20, Toluca, Mexique.

M.A. Laribi, A. Mlika, L. Romdhane and S. Zeghloul, 2004,"A Combined Genetic AlgorithmFuzzy Logic Method (GA-FL) in Mechanisms Synthesis", Mech. Mach. Theory 39, pp. 717-735.

Coxeter, H. S. M.1969, "Introduction to Geometry", 2nd ed. New York: Wiley.

Alden H. Wright, 1991,"Genetic algorithms for real parameter optimization, Foundations of Genetic Algorithms", (edited by Gregory J. E. Rawlins), Morgan Kaufman, pp. 205218.

Steiner, J., 1826 ,"Einige geometrische Betrachtungen." J. reine angew. Math. 1, pp. 161-184. 


\section{A Appendix}

The power of a fixed point $A$ (see Figure 17) with respect to a circle of radius $r$ and center $O$ is defined by the product

$$
f(A) \equiv A P \cdot A Q
$$

Where, $P$ and $Q$ are the intersections of a line through $A$ with the circle. The term "power" was first used in this way by Jacob Steiner [33,31]. $f(A)$ is independent of the choice of the line $A P Q$.

Now consider a point $A$ (see Figure 17) not necessarily on the circumference of the circle. If $d$ $=O A$ is the distance between $A$ and the circle's center $O$ with equation $f(x, y)=x_{2}+y_{2}-r_{2}=$ 0 , then the power of the point $A$ relative to the circle is givn by :

$$
f(A)=f\left(x_{A}, y_{A}\right)=x_{A}^{2}+y_{A}^{2}-r^{2}=d^{2}-r^{2}
$$

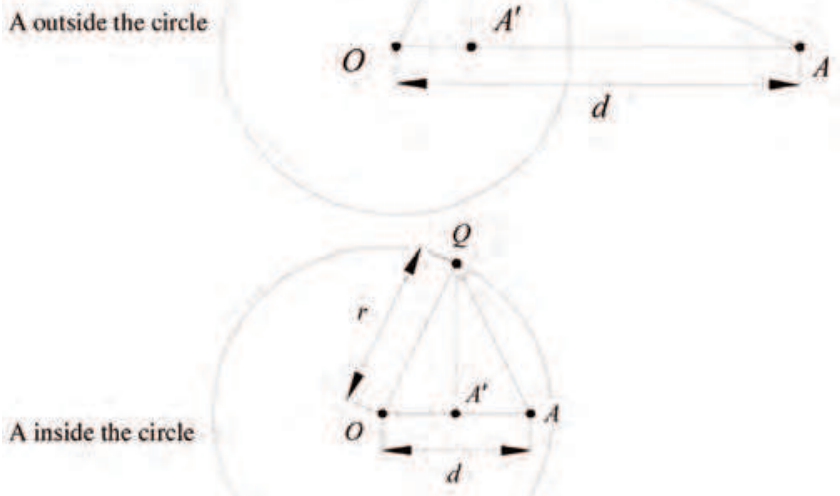

Fig. 17: The power of the point.

If $A$ is outside the circle, its power is positive and it is equal to the square of the length of the segment $A Q$ from $A$ to the tangent $Q$ to the circle through $A$,

$$
f(A)=A Q^{2}
$$

If $A$ is inside the circle, then the power is negative. 


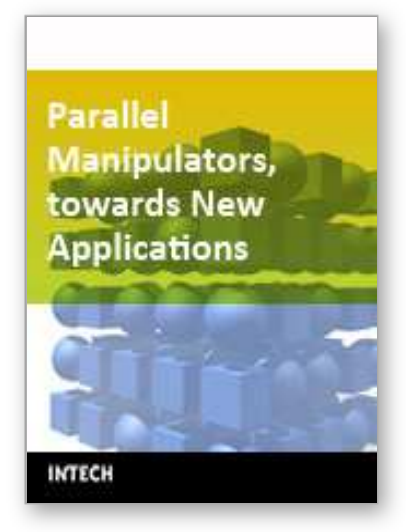

\author{
Parallel Manipulators, towards New Applications \\ Edited by Huapeng Wu
}

ISBN 978-3-902613-40-0

Hard cover, 506 pages

Publisher I-Tech Education and Publishing

Published online 01, April, 2008

Published in print edition April, 2008

In recent years, parallel kinematics mechanisms have attracted a lot of attention from the academic and industrial communities due to potential applications not only as robot manipulators but also as machine tools. Generally, the criteria used to compare the performance of traditional serial robots and parallel robots are the workspace, the ratio between the payload and the robot mass, accuracy, and dynamic behaviour. In addition to the reduced coupling effect between joints, parallel robots bring the benefits of much higher payload-robot mass ratios, superior accuracy and greater stiffness; qualities which lead to better dynamic performance. The main drawback with parallel robots is the relatively small workspace. A great deal of research on parallel robots has been carried out worldwide, and a large number of parallel mechanism systems have been built for various applications, such as remote handling, machine tools, medical robots, simulators, micro-robots, and humanoid robots. This book opens a window to exceptional research and development work on parallel mechanisms contributed by authors from around the world. Through this window the reader can get a good view of current parallel robot research and applications.

\title{
How to reference
}

In order to correctly reference this scholarly work, feel free to copy and paste the following:

M.A. Laribi, L. Romdhane and S. Zeghloul (2008). Advanced Synthesis of the DELTA Parallel Robot for a Specified Workspace, Parallel Manipulators, towards New Applications, Huapeng Wu (Ed.), ISBN: 978-3902613-40-0, InTech, Available from:

$\mathrm{http}: / /$ www.intechopen.com/books/parallel_manipulators_towards_new_applications/advanced_synthesis_of_t he_delta_parallel_robot_for_a_specified_workspace

\section{INTECH}

open science | open minds

\section{InTech Europe}

University Campus STeP Ri

Slavka Krautzeka 83/A

51000 Rijeka, Croatia

Phone: +385 (51) 770447

Fax: +385 (51) 686166

www.intechopen.com

\section{InTech China}

Unit 405, Office Block, Hotel Equatorial Shanghai

No.65, Yan An Road (West), Shanghai, 200040, China

中国上海市延安西路65号上海国际贵都大饭店办公楼405单元

Phone: +86-21-62489820

Fax: $+86-21-62489821$ 
(C) 2008 The Author(s). Licensee IntechOpen. This chapter is distributed under the terms of the Creative Commons Attribution-NonCommercialShareAlike-3.0 License, which permits use, distribution and reproduction for non-commercial purposes, provided the original is properly cited and derivative works building on this content are distributed under the same license. 